\title{
PROGRESS IN STUDY OF EFFICIENT CRYSTAL EXTRACTION AND COLLIMATION AT IHEP
}

\author{
A.G.Afonin, V.T.Baranov, V.M.Biryukov, V.N.Chepegin, Yu.A.Chesnokov, \\ Yu.S.Fedotov, V.I.Kotov, V.I.Terekhov, E.F.Troyanov - IHEP, Protvino, Russia; \\ Yu.M.Ivanov - PNPI, St. Petersburg, Russia; D.Trbojevic - BNL, USA; \\ W.Scandale - CERN, Switzerland; V.Guidi, G.Martinelli, M.Stefancich, \\ D.Vincenzi - Ferrara University, Italy; M.B.H.Breese - Surrey University, UK.
}

\begin{abstract}
The radical efficiency increase of accelerator beam extraction by means of bent crystals is reached by reducing the longitudinal dimension and bending angle of crystals. The usage of short crystals results in reduction of dechanneling losses and in increase of average number of crystal crossings by particle. Both factors allowed to get a $70 \mathrm{GeV}$ beam extraction efficiency more than $80 \%$ which was experimentally proved and is in a good agreement with theory predictions. The feasibility of efficient work of crystal at injection energy $(1.3 \mathrm{GeV})$ is demonstrated as well.
\end{abstract}

\section{ANALYSIS RESULTS}

New method of beam extraction from accelerator based on application of bent crystals [1-5] is developed in several world laboratories. This method is characterized by simplicity of realization, small intensity ripples and possibility to combine it with colliding mode or internal targeting. The crystal has minimum "septum thickness" and therefore can be very useful for loss localization applications as coherent scatterer.

However during a long time the researchers did not succeed to reach high efficiency of extraction, since at one passage of accelerator beam through a crystal only a small part of particles was captured into the channeling mode. The idea of significant increase of the extraction efficiency is to use a very short crystal [6,7]. In long crystals with large bending angle, used early, losses of particles were high due to dechanneling. In case of short crystals the growth of efficiency is reached because of a) reduction of particle losses caused by dechanneling, and $b$ ) increasing of average number of particle passages through crystal due to reduction of scattering on crystal length. The very first experimental work in this direction [3-5] performed by collaboration of researchers at IHEP $70 \mathrm{GeV}$ accelerator have resulted in essential improvement of extraction parameters in comparison with known data. Intensity of extracted $70 \mathrm{GeV}$ proton beam of more than $10^{11}$ per cycle was achieved at efficiency of $\sim 40 \%$. Two short silicon crystals ( length 7 and $5 \mathrm{~mm}$, bending angles 1.7 and $1.5 \mathrm{mrad}$ accordingly) made with two different technologies were used. One of the crystals had a shape of a strip and second had "O"figure shape (these designs are described in [3-5]).
These technologies of bent crystals preparation continue to be developed. The extraction efficiency up to $85 \%$ were achieved as a result of creation of new crystals, more shorter, better polished and better bent. At present a few crystals are installed at the U-70 accelerator. Their location are chosen so that the bent crystals are used as the first elements of slow extraction system. The characteristics of crystals are given in Table 1. The crystals shaped as a strip (S-type) have orientation $\mathrm{Si}(111)$, while the O-shaped crystals have the orientation $\operatorname{Si}(110)$.

Steering the accelerator beam onto a crystal is provided by slow bump distorting the orbit. Location of the equipment and diagnostics devices are described in detail in [3-5] as well as the characteristics of beam in the accelerator.

Table 1. Crystal characteristics

\begin{tabular}{|c|c|c|c|c|c|}
\hline 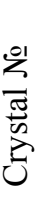 & 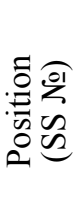 & 总 & 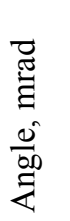 & 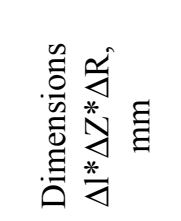 & 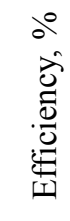 \\
\hline \multicolumn{6}{|c|}{ EXTRACTION } \\
\hline 1 & 106 & $\mathrm{~S}$ & 1.0 & $2.0 \times 35 \times 0.5$ & 85 \\
\hline 2 & 106 & $\mathrm{O}$ & 0.7 & $3.5 \times 5.0 \times 0.7$ & 60 \\
\hline 3 & 19 & $\mathrm{~S}$ & 2.0 & $5.0 \times 45 \times 0.5$ & 67 \\
\hline 4 & 19 & $\mathrm{O}$ & 2.1 & $5.0 \times 5.0 \times 0.7$ & 65 \\
\hline 5 & 19 & $\mathrm{O}$ & 2.3 & $5.0 \times 5.0 \times 0.6$ & 45 \\
\hline \multicolumn{6}{|c|}{ COLLIMATION } \\
\hline 6 & 84 & $\mathrm{~S}$ & 0.8 & $1.8 \times 27 \times 0.5$ & $\begin{array}{c}85 \\
20^{*}\end{array}$ \\
\hline 7 & 84 & $\mathrm{O}$ & 1.7 & $2.5 \times 5.0 \times 0.5$ & 60 \\
\hline 8 & 86 & S & 1.4 & $4.0 \times 45 \times 0.5$ & 65 \\
\hline
\end{tabular}

All devices of the beam diagnostics were tested and calibrated with the fast extracted beam. The absolute error of intensity measurements of extracted beam did not exceed 2\% [8]. The background conditions were periodically measured by disorientation of the crystal. According to measurements, the level of a background together with noise of the equipment did not exceed 3\% of channeled beam intensity. The fraction of the beam 
steered on the crystal was determined with the systematic error $\sim 1 \%$ by measurements of circulating beam intensity before and after extraction. Total systematic error of efficiency measurements was $\sim 4 \%$ taking into account all the factors. Efficiency of extraction (ratio of extracted beam intensity to the accelerator intensity steered onto the crystal) was determined at each accelerator cycle. The statistics for several hundreds of cycles was stored for each experimental point. In order to get uniform steering of the beam onto a crystal a feedback loop including secondary particles monitor and bump power supply was used.

The best result was achieved using shortest crystals №1 and № 6 with lengths of $2 \mathrm{~mm}$ and $1.8 \mathrm{~mm}$ shaped as narrow strips.

The results of investigations of crystal №1 are given on Fig. 1and 2. With this crystal the extraction efficiency of $(85 \pm 2.8) \%$ was achieved with accelerator beam intensity

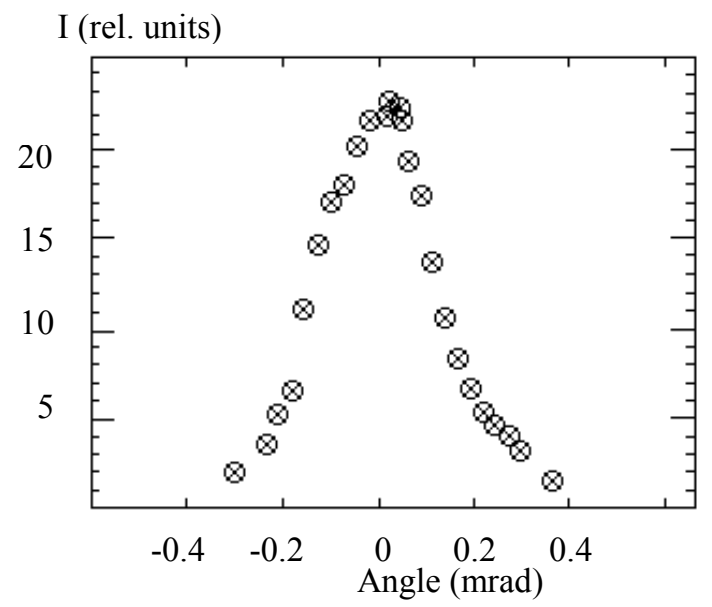

Figure 1. Orientation dependence of extracted intensity.

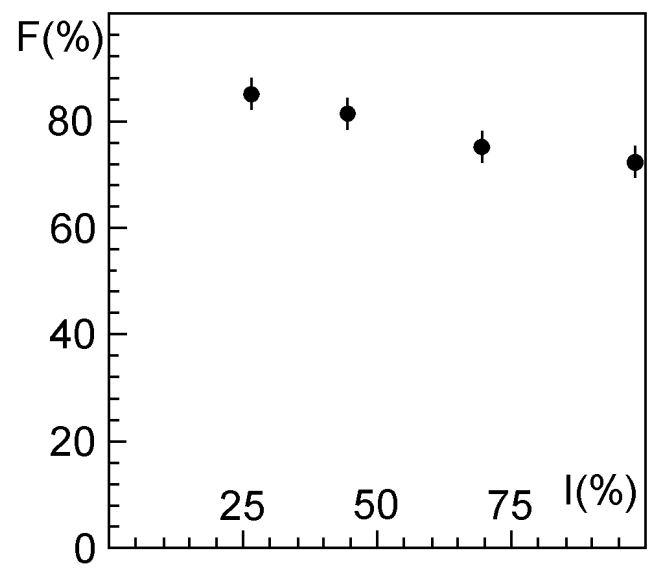

Figure 2. Extraction efficiency as a function of the fraction of incident beam.

of $1 * 10^{12}$ particles per cycle. Further investigations will show whether the intensity of the beam extracted by crystal can be increased.
The crystal №6 was used in a system of loss localization as a coherent scatterer. It was installed $20 \mathrm{~m}$ upstream the beam collimator. The crystal deflected $(85 \pm 2.8) \%$ of steered particles inside the collimator body. Fig. 3 presents results of beam profile measurement at collimator entry in different modes.

Steering the beam directly on collimator edge (3a)
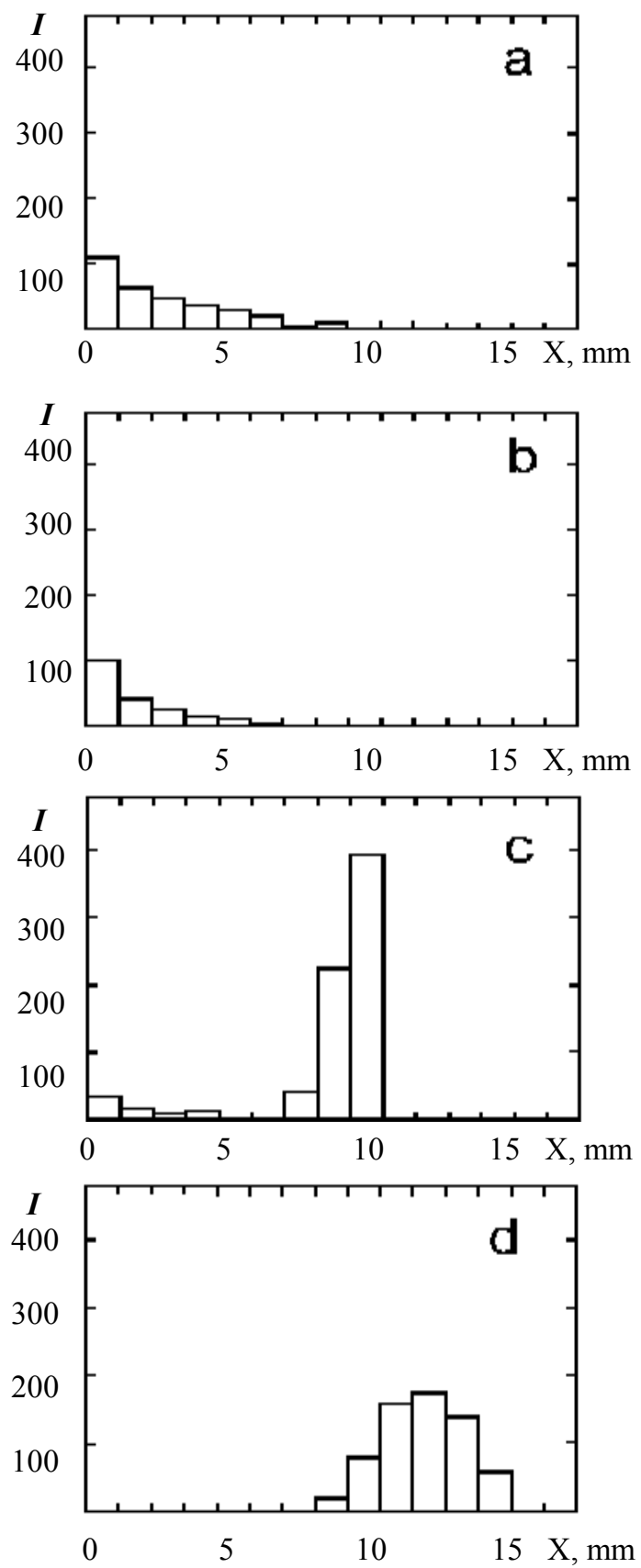

Figure 3. $70 \mathrm{GeV}$ beam profiles at collimator entry face measured in different modes.

results in concentration of the particles near the edge as expected. This reduces efficiency of collimation. The case (3b) corresponds to disoriented crystal while the case (3c) corresponds to oriented crystal, when the large part of particles is deflected deep into the collimator. The plot (3d) reflects a situation, when a beam 
is directed on collimator by a fast kicker-magnet. The last mode was also applied for calibration of devices used for crystal operation diagnostics. Application of a crystal reduces the radiation levels downstream the collimator in several times.

Fig. 4 summarizes experimental results of extraction efficiency measurements for the crystals of a different length (Table 1). These results are compared with the calculated ones, taking into account transportation of particles in a crystal (program CATCH [9]) and multiturn movement in accelerator. As it can be seen on this figure, the experimental data are in good agreement with the simulation.

The short crystals with a length of $\sim 1 \mathrm{~mm}$ along the beam can be applied not only for high energy accelerators, but also at energy of particles $\sim 1 \mathrm{GeV}$. Thus, the first tests of $1.3 \mathrm{GeV}$ protons deflection were carried out at injection plateau of U-70 accelerator. The

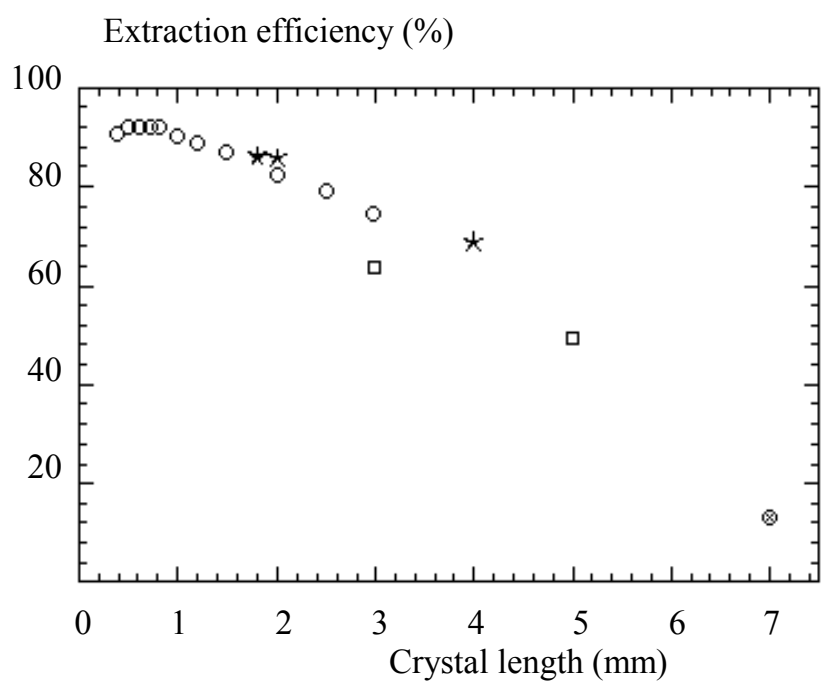

Figure 4. Crystal extraction efficiency:

$$
\begin{array}{ll}
\star, \otimes & : \text { strips; } \\
\square & : \text { O - shape; } \\
\bigcirc & : \text { ideal strip, predicted [11] }
\end{array}
$$

profile of the beam deflected by crystal №6 under these conditions is shown on Fig. 5. Specific feature of this case is significant multiple scattering of particles by a crystal. RMS angle of scattering equal to $\sim 1 \mathrm{mrad}$ is comparable with the angle of crystal bend. However even under these conditions near $50 \%$ of the particles brought on collimator are channeled particles. The corresponding extraction efficiency of channeled beam in this case is $\sim 20 \%$ according to estimations. The further optimization of short crystals, using the new technology of their growing [10], may allow in principle to reach extraction efficiency for low energies (less than $1 \mathrm{GeV}$ ) as high as it was achieved for $70 \mathrm{GeV}$.

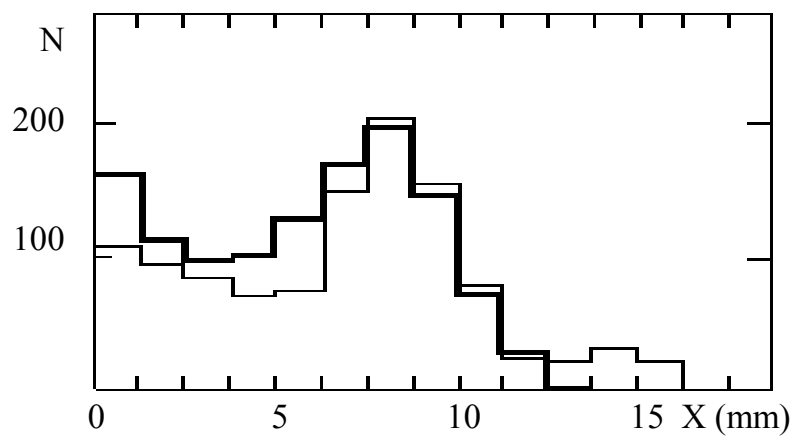

Figure 5. $1.3 \mathrm{GeV}$ beam profile at collimator entry face: measured (thick) and calculated (thin).

The application of crystal extraction at IHEP accelerator for experimental setup for study the rare decays of K-mesons has allowed to increase in 40 times world statistics of these decays during 2 months of accelerator operation [11]. Integral flux of particles crossed the crystal during this time was $2 * 10^{20} / \mathrm{cm}^{2}$. It should be noted, that in this case the crystal extraction of protons worked simultaneously with two internal targets generating the secondary particles for other experiments.

Results of investigations show, that the bent crystals can be successfully applied to extraction and collimation of beams on accelerators in a wide range of energies.

The work was supported by grants RFBR 01-02-16229 and INTAS-CERN132-2000.

\section{REFERENCES}

[1] H. Akbari, X. Altuna, S. Bardin et al. Phys. Lett. B 313 (1993), 491.

[2] C.T.Murphy, R.A.Carrigan, Jr. D.Chen et al. Nucl. Instr. and Meth B 119 (1996), 231.

[3] A.G.Afonin, V.M.Biryukov, V.A.Gavrilushkin et al. JETP Lett., 67 (1998), 781.

[4] A.A.Arkhipenko, A.G.Afonin, B.I.Baranov et al. Phys. Lett. B 435 (1998), 240.

[5] A.G.Afonin, V.M.Biryukov, V.A.Gavrilushkin et al. JETP Lett., 568 (1998).

[6] V.Biryukov, Nucl. Instr. and Meth. B53 (1991), 202.

[7] A.M. Taratin, S.A.Vorobiev, M.D. Bavizhev et al. Nucl. Instr. and Meth. B 58 (1991), 103.

[8] A.G.Afonin, V.N.Gres and V.I.Terekhov, Proc. of EPAC (Stockholm, 1998), 1613.

[9] V.Biryukov. Phys. Rev. E 51 (1995), 3522.

[10] M.Breese. Nucl. Instr. and Meth. 132, 540 (1997).

[11] V.I.Kotov, A.G.Afonin, V.M.Biryukov et al. Proc. of EPAC (Vienna, 2000), 364. 